\title{
A Patient with Metastatic Lung Cancer and Dysphagia
}

Biechele, Judith ; Fox, Mark

DOI: https://doi.org/10.1053/j.gastro.2019.10.047

Posted at the Zurich Open Repository and Archive, University of Zurich ZORA URL: https://doi.org/10.5167/uzh-184485

Journal Article

Accepted Version

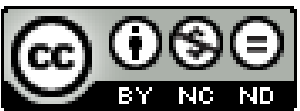

The following work is licensed under a Creative Commons: Attribution-NonCommercial-NoDerivatives 4.0 International (CC BY-NC-ND 4.0) License.

Originally published at:

Biechele, Judith; Fox, Mark (2020). A Patient with Metastatic Lung Cancer and Dysphagia. Gastroenterology, 158(3):485-487.

DOI: https://doi.org/10.1053/j.gastro.2019.10.047 


\section{CLINICAL CHALLENGES AND IMAGES IN GI}

\section{A patient with metastatic lung cancer and dysphagia}

\section{Authors}

Judith Biechele 1, Mark Fox 2,3

\section{Affiliations}

${ }^{1}$ Department of Oncology, Klinik Arlesheim, Arlesheim, Switzerland

${ }^{2}$ Department of Gastroenterology and Hepatology, University Hospital Zurich, Zurich, Switzerland

${ }^{3}$ Digestive Function: Basel, Laboratory and Clinic for motility disorders and functional GI diseases, Center for integrative Gastroenterology, Klinik Arlesheim, Arlesheim, Switzerland

\section{Corresponding author contacts}

Digestive Function: Basel, Laboratory and Clinic for motility disorders and functional GI diseases, Center for integrative Gastroenterology, Klinik Arlesheim, Arlesheim, Switzerland; Email: dr.mark.fox@gmail.com

Conflict of interest statement: neither of the authors report any conflicts of interest

\section{Question}

A 73-year-old woman with advanced lung cancer presented to our clinic with increasing pain, weakness and weight loss related to metastatic disease involving the liver, lymphatic system and skeleton. The patient also complained of increasing difficulty with eating over several weeks. Initially, her symptoms were worse with solids, but progressed to involve ingestion of pureed food and yoghurt. Constipation was a problem as well.

Adeno-carcinoma of the lung had been diagnosed by imaging and biopsy of a liver metastasis in February 2019. A palliative chemotherapy regime with Cisplatin/Pemetrexed and Binetimib was commenced in April 2019; however, staging investigations in July 2019 showed disease progression under treatment. Test results also raised concerns that a second cancer in the gastrointestinal tract may be present because of increasing anemia $(\mathrm{Hb} 70 \mathrm{~g} / \mathrm{l})$, an elevated CEA (54.3ng/l), and CA125 (3219U/ml).

The patient decided against continuation of the chemotherapy. Best supportive care was instituted. This included a step-wise increase in opioid therapy with Oxycodon/Naloxon 
20/10mg twice daily with additional oral morphine as required. Constipation was treated with osmotic laxatives (Transipeg, Magnesium). Further complementary, anthroposophic medicine including rhythmic massage and external application of compresses were applied to relieve her abdominal symptoms.

During her admission, the patient's swallowing difficulties became acute. She could swallow only a few mouthfuls of food or drink before « vomiting » undigested food and secretions. Physical examination revealed a cachectic woman with BMI of $17.7 \mathrm{~kg} / \mathrm{m}^{2}$. There were dry mucous membranes in the mouth, but no sign of candida infection. The oro-pharyngeal swallow was intact. The abdomen was soft, slightly tender to palpation with no masses and few belly sounds. Rectal digital examination showed no fecal impaction and an abdominal X-ray showed no sign of ileus. Laboratory testing revealed no additional abnormalities.

Endoscopic examination and cross-sectional imaging did not show obstruction or other pathology of the oesophagus or mediastinum that could explain the dysphagia (figure 1A/B). However, endoscopic functional luminal impedance planimetry (EndoFLIP ${ }^{\circledR}$; Medtronic) suggested reduced distensibility during balloon distension. Due to the severity of the symptoms and their impact on her quality of life an esophageal high-resolution manometry (HRM) was performed to clarify the diagnosis. This investigation demonstrated premature, simultaneous contractions with a short Distal Latency (DL $<4.5 \mathrm{~s}$ ) and abnormal esophago-gastric junction (EGJ) function with pathological integrated relaxation pressure (IRP >15 $\mathrm{mmHg}$ ) well above normal levels (figure 1C). A specific treatment was implemented. Two weeks later the patient was able to eat most foods without difficulty, EGJ distensibility had increased on EndoFLIP ${ }^{\circledR}$ and esophageal motility was normal on repeat HRM investigation (figure 1D).

\section{Question: What is the diagnosis and what treatment(s) could have been applied?}

\section{Answer to: Image 1 (Page YYY):}

The esophageal HRM shows achalasia type III defined by the presence of abnormal EGJ function and premature, spastic contractions by the Chicago Classification of oesophageal motility disorders (Figure 1C). This finding is consistent also with reduced EGJ distensibility on EndoFLIP investigation (Figure 1C). Outlet obstruction by metastases from the primary lung cancer (or a second carcinoma) at the EGJ had been excluded by endoscopy and imaging (Figure 1A/B). Pseudo-achalasia caused by circulating antibodies to receptors that mediate deglutitive relaxation of the esophagus and EGJ was possible; however, this is a very rare condition. Another cause of a severe motility disorder in a patient recently prescribed increasing doses of opioid analgesia is Opioid-Induced Esophageal Dysfunction (OIED). 
It was not possible to reduce the dose of opioid analgesia in this patient; indeed the dose was increased to MST continuous $60 \mathrm{mg}$ twice daily following the diagnosis of achalasia. The risk of perforation from endoscopy with pneumatic dilatation of the EGJ was considered to be high in this cachectic patient and the efficacy of botulinum toxin for the management of achalasia is suboptimal. Taking all these factors into account, a therapeutic trial of Naloxegol (Moventig, AstraZeneca), a long-acting peripherally acting mu-opioid receptor antagonist (PAMORA), was commenced to treat suspected OIED. Because the patient reported no side effects and no reduction in analgesia on this medication, the initial dose of $12.5 \mathrm{mg}$ was increased in a step-wise manner to 50mg once daily (the maximum dose). As the dose of Naloxegol was increased, the patient started to eat again with much reduced dysphagia and volume regurgitation. Having been able to take only sips of nutrient drinks before this treatment commenced; now she could eat soft solid foods without difficulty. The patient consented to a repeat HRM 16 days after the initial diagnosis. This demonstrated that the improvement in swallowing function was accompanied by a remarkable return to normal esophageal motility and EGJ distensibility (figure 1D). Together, the improvement in clinical condition and HRM findings on the peripheral opioid antagonist confirmed the diagnosis of OIED.

Patients taking opioid analgesics often complain about gastrointestinal side effects, in particular constipation, due to activation of peripheral opioid receptors, which can cause abnormal visceral motility, secretion and sensation. To manage these problems, oral preparations are available that combine strong opioids with the short-acting opioid antagonist Naloxone (Targin, Purdue Pharma) that does not cross the blood-brain barrier. Recently once daily dosing with long-acting Naloxegol (AstraZeneca) has been approved by regulatory bodies for the treatment of opioid-induced constipation (OIC) in patients with chronic pain syndromes.

Recently it has been recognized that opioid treatment is also a cause of esophageal symptoms including dysphagia, reflux symptoms and chest pain. Case series from the Mayo Clinic in Scottsdale Arizona report that approximately 1 in 4 patients taking opioids referred for HRM have a major motility disorder compared to 1 in 10 patients overall.[1,2] Patients on these medications have higher prevalence of achalasia, EGJ outflow obstruction, distal esophageal spasm and jackhammer esophagus.[1,2] The prevalence of OIED is dose-dependent and more common in patients taking strong opioids (e.g. oxycodone) compared to tramadol (31\% vs. 12\%)).[2] There is also an increase in OIED for patients who were studied on opioids (last dose within 24 hours of HRM), compared to chronic users studied off opioids (last dose $\geq 24$ hours from HRM) (33\% vs. 15\%).[1, 2]

Because the mechanism underlying the effects of opioids on esophageal motility have not been fully clarified, it can be hard to differentiate between primary, idiopathic achalasia and 
secondary, iatrogenic disease. Physiological and clinical studies have shown that patients with OIED have different responses to amyl nitrite and cholecystokinin.[3] Based on these observations it has been suggested that opioids inhibit nitric oxide releasing neurons in the esophagus allowing unopposed excitatory input from cholinergic neurons leading to the impaired EGJ relaxation, and premature, spastic esophageal contractions.

With the exception of dose-reduction or withdrawal of opioids there is no established treatment for OIED. Furthermore, conventional therapies (pneumatic dilatation or myotomy) that are known to be effective for achalasia have disappointing results in this patient group. Given the difficulty in reducing analgesic medication in patients with chronic pain, it is appropriate to consider the use of peripheral opiate antagonists such as naloxegol in the management of OIED. To our knowledge, this is the first case report of the successful use of naloxegol (or any PAMORA) in this condition. Our experience indicates that this medication can provide effective relief of symptomatic OIED in a palliative situation, where opioids cannot easily be withdrawn. Pneumatic dilatation, surgical myotomy, or per-oral endoscopic myotomy should be reserved for refractory cases and should be pursued with caution, after review of risks and benefits with the patients given reports of lower response rates in this patient group.

1. Ratuapli, S.K., et al., Opioid-Induced Esophageal Dysfunction (OIED) in Patients on Chronic Opioids. Am J Gastroenterol, 2015. 110(7): p. 979-84.

2. Snyder, D.L., et al., Opioid-Induced Esophageal Dysfunction: Differential Effects of Type and Dose. Am J Gastroenterol, 2019. 114(9): p. 1464-1469.

3. Babaei, A., S. Shad, and B.T. Massey, Motility Patterns Following Esophageal Pharmacologic Provocation With Amyl Nitrite or Cholecystokinin During High-resolution Manometry Distinguish Idiopathic vs Opioid-induced Type 3 Achalasia. Clin Gastroenterol Hepatol, 2019. 

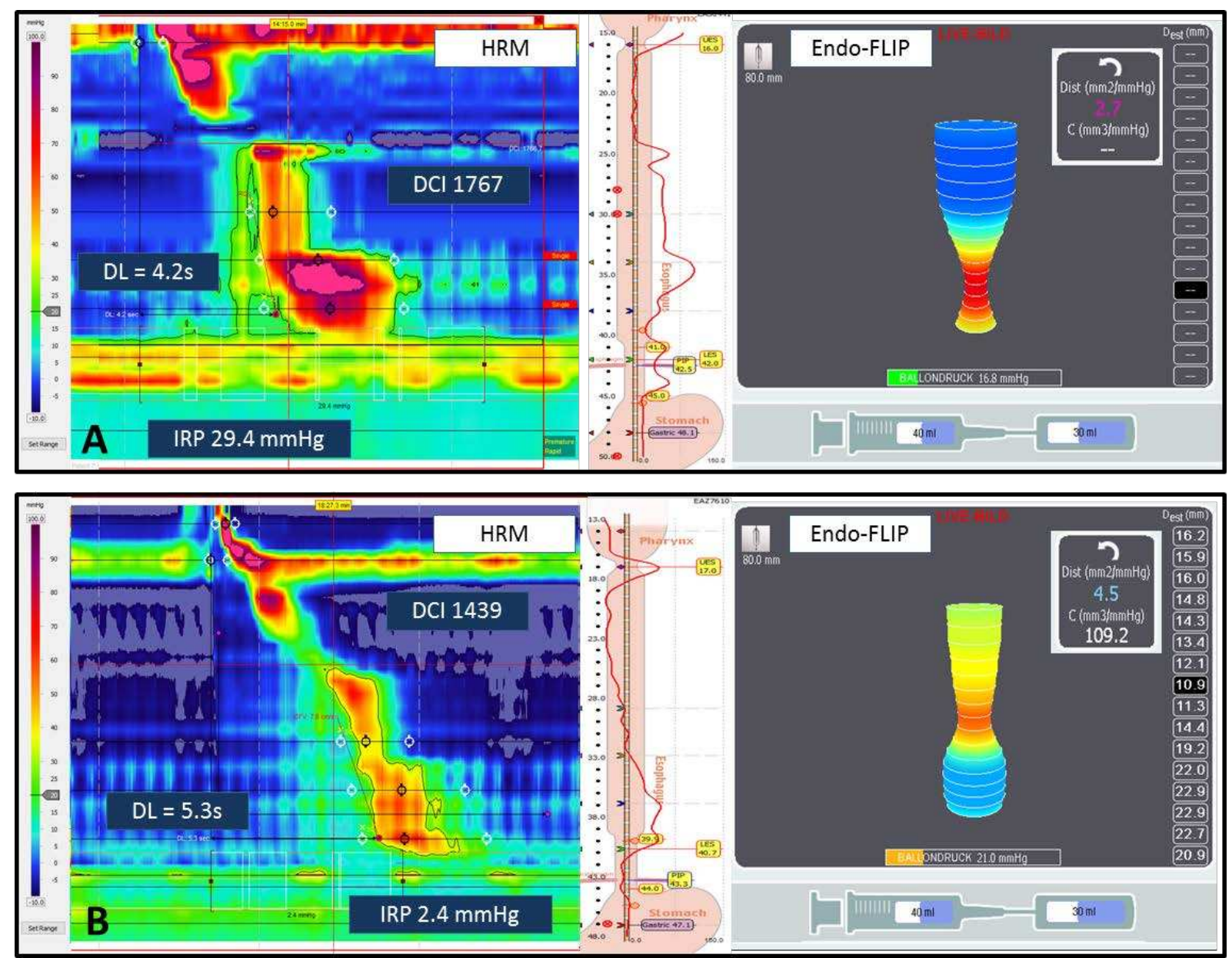